Article

\title{
Computing The Irregularity Strength of Planar Graphs
}

\author{
Hong Yang ${ }^{1}$, Muhammad Kamran Siddiqui ${ }^{2, * \mathbb{D}}$, Muhammad Ibrahim ${ }^{3}$ and Sarfraz Ahmad ${ }^{4}$ \\ and Ali Ahmad ${ }^{5}$ \\ 1 School of Information Science and Engineering, Chengdu University, Chengdu 610106, China; \\ yanghong01@cdu.edu.cn \\ 2 Department of Mathematics, COMSATS University Islamabad, Sahiwal Campus, Sahiwal 57000, Pakistan \\ 3 Center for Advanced Studies in Pure and Applied Mathematics, Bahauddin Zakariya University Multan, \\ Multan 60800, Pakistan; mibtufail@gmail.com \\ 4 Department of Mathematics, COMSATS University Islamabad, Lahore Campus, Lahore 54000, Pakistan; \\ sarfrazahmad@cuilahore.edu.pk \\ 5 College of Computer Science \& Information Systems, Jazan University, Jazan 45142, Saudi Arabia; \\ ahmadsms@gmail.com \\ * Correspondence: kamransiddiqui75@gmail.com
}

Received: 23 July 2018; Accepted: 27 August 2018; Published: 30 August 2018

\begin{abstract}
The field of graph theory plays a vital role in various fields. One of the important areas in graph theory is graph labeling used in many applications such as coding theory, $X$-ray crystallography, radar, astronomy, circuit design, communication network addressing, and data base management. In this paper, we discuss the totally irregular total $k$ labeling of three planar graphs. If such labeling exists for minimum value of a positive integer $k$, then this labeling is called totally irregular total $k$ labeling and $k$ is known as the total irregularity strength of a graph $G$. More preciously, we determine the exact value of the total irregularity strength of three planar graphs.
\end{abstract}

Keywords: total edge irregularity strength; total vertex irregularity strength; total irregularity strength; planar graph

MSC: 05C12; 05C78; 05C90

\section{Introduction}

All graphs considered here are finite, undirected, without loops or multiple edges. Denote by $V(G)$ and $E(G)$ the set of vertices and the set of edges of a graph $G$, respectively. Let $|V(G)|=n$ and $|E(G)|=m$.

A labeling of a graph is any mapping that sends some set of graph elements to a set of numbers or colors. Graph labeling provides valuable information used in several application areas (see [1]). It is interesting to consider labeling the elements of the graph by the elements of a finite field.

For a graph $G$, we characterize a labeling $\zeta: V \cup E \rightarrow\{1,2, \ldots, k\}$ to be total $k$-labeling. A total $k$-labeling is characterized to be an edge irregular total $k$-labeling of the graph $G$ if for each two distinct edges $r s$ and $r^{\prime} s^{\prime}$ their weights $\phi(r)+\phi(r s)+\phi(s)$ and $\phi\left(r^{\prime}\right)+\phi\left(r^{\prime} s^{\prime}\right)+\phi\left(s^{\prime}\right)$ are distinct. In addition, total $k$-labeling is characterized to be a vertex irregular total $k$-labeling of the graph $G$ if for each two distinctive vertices $r$ and $s$ their weights $w t(r)$ and $w t(s)$ are distinct. Here, the weight of a vertex $r$ in $G$ is the sum of the label of $r$ and the labels of all edges incident with the vertex $r$. The least $k$ for which the graph $G$ has an edge irregular total $k$-labeling is called the total irregularity strength of $G$, represented by tes $(G)$. Analogously, the minimum $k$ for which the graph $G$ has a vertex irregular total $k$-labeling is called the total vertex irregularity strength of $G$, denoted by $\operatorname{tvs}(G)$. 
Chartrand et al. [2] introduced two graph invariants namely irregular assignments and the irregularity strength. Baca et al. [3] modified these graph invariants and introduced the concept of total edge irregularity strength and total vertex irregularity strength for a graph $G$. A simple lower bound for tes $(G)$ and $\operatorname{tus}(G)$ of a $(p, q)$-graph $G$ in terms of maximum degree $\Delta(G)$ and the minimum degree $\delta(G)$, determine in the following theorems.

Theorem 1. [3] Let $G$ be a finite graph with $p$ vertices, $q$ edges and having maximum degree $\Delta=\Delta(G)$, the upper square brackets represent the ceiling function, and then

$$
\operatorname{tes}(G) \geq \max \left\{\left\lceil\frac{q+2}{3}\right\rceil,\left\lceil\frac{\Delta+1}{2}\right\rceil\right\}
$$

Theorem 2. [3] Let $G$ be a finite graph with $p$ vertices, q edges, minimum degree $\delta=\delta(G)$ and maximum degree $\Delta=\Delta(G)$, the upper square brackets represent the ceiling function, and then

$$
\left\lceil\frac{p+\delta}{\Delta+1}\right\rceil \leq \operatorname{tvs}(G) \leq p+\Delta-2 \delta+1
$$

In [4], Ivančo and Jendrol' posed the following conjecture:

Conjecture 1. [4] Let $G$ be a finite graph with $p$ vertices, $q$ edges, different from $K_{5}$ with minimum degree $\delta=\delta(G)$, maximum degree $\Delta=\Delta(G)$, the upper square brackets represent the ceiling function, and then

$$
\operatorname{tes}(G)=\max \left\{\left\lceil\frac{q+2}{3}\right\rceil,\left\lceil\frac{\Delta+1}{2}\right\rceil\right\}
$$

In [5], Nurdin et al. posed the following conjecture:

Conjecture 2. [5] Let $G$ be a connected graph having $n_{i}$ vertices of degree $i(i=\delta, \delta+1, \delta+2, \ldots, \Delta)$, where $\delta$ and $\Delta$ are the minimum and the maximum degree of $G$, respectively. Moreover, the upper square brackets represent the ceiling function, and then

$$
\operatorname{tvs}(G)=\max \left\{\left\lceil\frac{\delta+n_{\delta}}{\delta+1}\right\rceil,\left\lceil\frac{\delta+n_{\delta}+n_{\delta+1}}{\delta+2}\right\rceil, \ldots,\left\lceil\frac{\delta+\sum_{i=\delta}^{\Delta} n_{i}}{\Delta+1}\right\rceil\right\} .
$$

Conjecture 1 has been shown for complete graphs and complete bipartite graphs [6,7], for hexagonal grid graphs [8], for toroidal grid [9], for generalized prism [10], for strong product of cycles and paths [11], for categorical product of two cycles [12], for zigzag graphs [13] and for strong product of two paths [14].

Conjecture 2 has been verified for for circulant graphs [15].

Combining both total edge irregularity strength and total vertex irregularity strength notions, Marzuki et al. [16] introduced a new irregular total $k$-labeling of a graph $G$, which is required to be at the same time both vertex and edge irregular as follows:

Definition 1. A total labeling $\phi: V \cup E \rightarrow\{1,2, \ldots, k\}$ is called totally irregular total $k$-labeling of $G$ if every two distinct vertices $u$ and $v$ in $V(G)$ satisfy $w t(u) \neq w t(v)$, and every two distinct edges $u_{1} u_{2}$ and $v_{1} v_{2}$ in $E(G)$ satisfy $w t\left(u_{1} u_{2}\right) \neq w t\left(v_{1} v_{2}\right)$, where $w t(u)=\phi(u)+\sum_{u v \in E(G)} \phi(u v)$ and $w t\left(u_{1} u_{2}\right)=\phi\left(u_{1}\right)+\phi\left(u_{1} u_{2}\right)+\phi\left(u_{2}\right)$. The minimum $k$ for which a graph $G$ has a totally irregular total $k$-labeling is called the total irregularity strength of $G$, denoted by $t s(G)$. 
Marzuki, et al. [16] gave a lower bond of $t_{s}(G)$ as follows:

$$
\text { For every graph } G, \operatorname{ts}(G) \geq \max \{\operatorname{tes}(G), \operatorname{tvs}(G)\}
$$

Ramdani and Salman [17] showed that the lower bound in Equation (1) for some cartesian product graphs is tight. Besides that, they determined the total irregularity strength of cycles and paths. For more details, see [18-20]. In [21], Ahmad et al. found the exact value of total irregularity strength of generalized Petersen graph.

Example 1. For illustration, the concept of the totally irregular total k-labeling, we give an example from our recent paper [21] in which we show the totally irregular total 10-labeling for generalized Petersen graph $P(9,2)$ (see Figure 1).

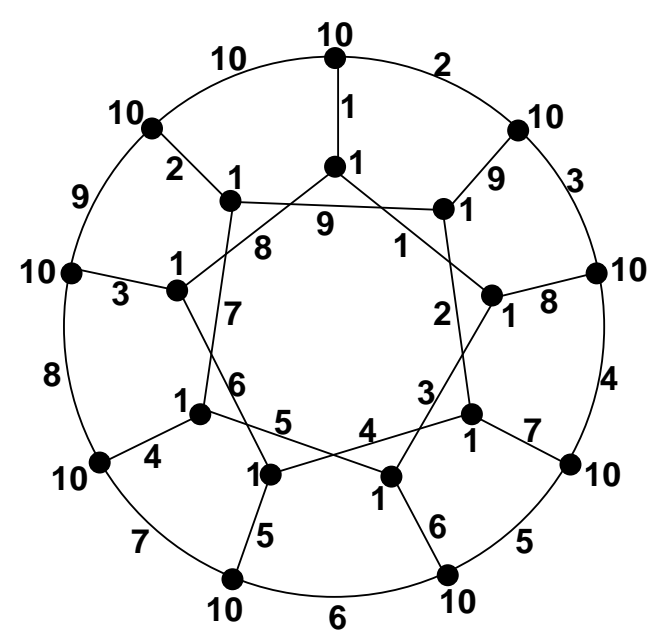

Figure 1. A totally irregular total 10-labeling for $P(9,2)$.

The weights for all vertices and the weights for all edges under the totally irregular total 10-labeling are given in Figure 2.

Now, from Figure 2, it is easy to check that edge weights are different and represented by blue. On the other hand, the vertex weights are different and represented by black.

In this paper, we investigate the total irregularity strength of planar graphs.

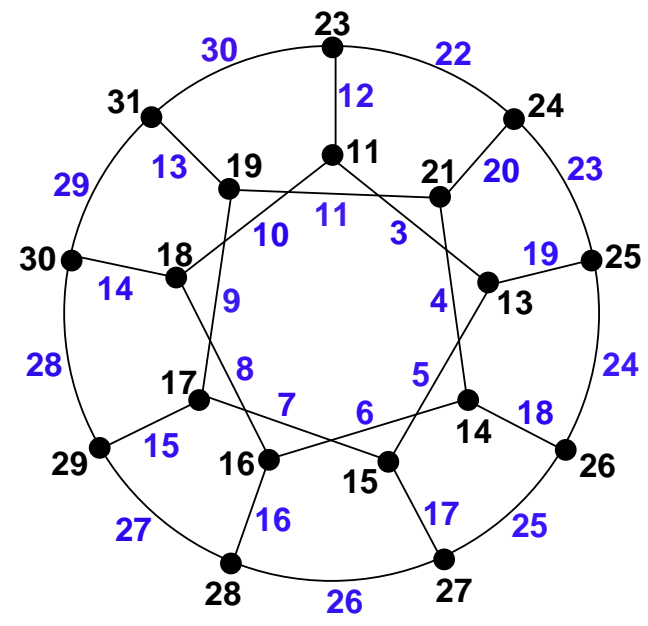

Figure 2. The weights of vertices and edges for $P(9,2)$. 


\section{The Planar Graph $T_{n}$}

Siddiqui introduced the planar graph $S_{n}$ in [22] and computed the $t e s\left(S_{n}\right), \operatorname{tvs}\left(S_{n}\right)$. The planar graph $T_{n}$ (see Figure 3) is obtained from the planar graph $S_{n}$ by adding new edges $x_{i} y_{i+1}$ and having the same vertex set. The planar graph $T_{n}$ has

$$
\begin{gathered}
V\left(T_{n}\right)=\left\{x_{i} ; y_{i} ; z_{i} ; 1 \leq i \leq n\right\} \\
E\left(T_{n}\right)=\left\{z_{i} z_{i+1} ; y_{i} y_{i+1}: 1 \leq i \leq n\right\} \cup\left\{x_{i} y_{i} ; y_{i} z_{i} ; x_{i} y_{i+1} ; y_{i+1} z_{i}: 1 \leq i \leq n\right\}
\end{gathered}
$$

Clearly, the planar graph $T_{n}$ has $3 n$ vertices and $6 n$ edges. More preciously, we call the cycle induced by $\left\{z_{i}: 1 \leq i \leq n\right\}$ the inner cycle, cycle induced by $\left\{y_{i}: 1 \leq i \leq n\right\}$ the outer cycle, and the set vertices $\left\{x_{i}: 1 \leq i \leq n\right\}$, the outer vertices. All subscripts are taken under modulo $n$. In the next theorem, we determine the total irregularity strength of the planar graph $T_{n}$.
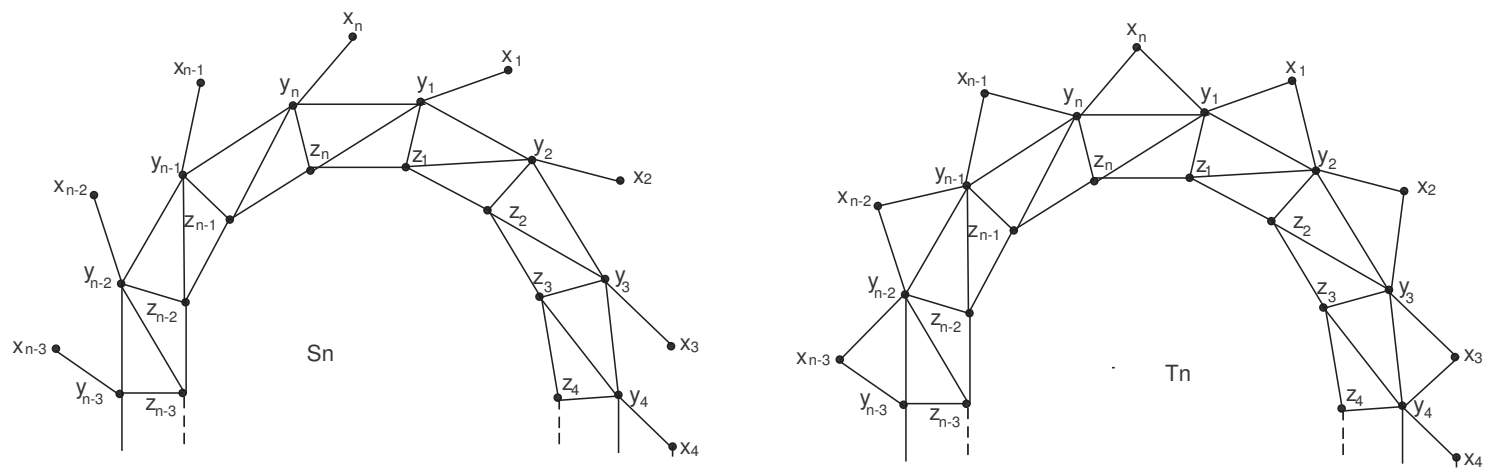

Figure 3. The planar graph $S_{n}$ and $T_{n}$.

Theorem 3. Let $T_{n}, n \geq 3$ be a planar graph. Then, $t s\left(T_{n}\right)=\left\lceil\frac{6 n+2}{3}\right\rceil$.

Proof. Since $\left|E\left(T_{n}\right)\right|=6 n$, from Theorem 1 , tes $\left(T_{n}\right) \geq\left\lceil\frac{6 n+2}{3}\right\rceil$. In addition, $T_{n}$ has $n$ vertices of degree $2, n$ vertices of degree 4 , and $n$ vertices of degree 6; thus, from Theorem 2, we get $\operatorname{tvs}\left(T_{n}\right) \geq\left\lceil\frac{2 n+2}{4}\right\rceil$. From Equation (1), we get $t_{s}\left(T_{n}\right) \geq\left\lceil\frac{6 n+2}{3}\right\rceil$. Now, we show that $t_{s}\left(T_{n}\right) \leq\left\lceil\frac{6 n+2}{3}\right\rceil$. For this, we define a total labeling $\phi$ from $V\left(T_{n}\right) \cup E\left(T_{n}\right)$ into $\left\{1,2, \ldots,\left\lceil\frac{6 n+2}{3}\right\rceil\right\}$ and compute the vertex weight and edge weights in the following way.

Let $k=\left\lceil\frac{6 n+2}{3}\right\rceil$. For $1 \leq i \leq n$, we have $\phi\left(x_{i}\right)=\phi\left(y_{i}\right)=i, \phi\left(z_{i}\right)=k, \phi\left(x_{i} y_{i}\right)=1, \phi\left(y_{i} y_{i+1}\right)=k+1-i, \phi\left(y_{i} z_{i}\right)=n+1, \phi\left(y_{i+1} z_{i}\right)=k$, $w t\left(x_{i} y_{i}\right)=2 i+1, w t\left(x_{i} y_{i+1}\right)=2 i+2, w t\left(y_{i} z_{i}\right)=k+n+1+i, w t\left(y_{i}\right)=k+5 n+8-i$,

$$
\begin{gathered}
\phi\left(x_{i} y_{i+1}\right)= \begin{cases}1, & \text { for } 1 \leq i \leq n-1 \\
n+1, & \text { for } i=n\end{cases} \\
w t\left(y_{i} y_{i+1}\right)= \begin{cases}k+2+i, & \text { for } 1 \leq i \leq n-1 \\
k+2, & \text { for } i=n\end{cases}
\end{gathered}
$$




$$
\begin{gathered}
\phi\left(z_{i} z_{i+1}\right)= \begin{cases}k+1-i, & \text { for } 1 \leq i \leq n-3 \\
n+3, & \text { for } i=n-2 \\
n+4, & \text { for } i=n-1 \\
n+1, & \text { for } i=n, n \text { is even } \\
n+2, & \text { for } i=n, n \text { is odd }\end{cases} \\
w t\left(y_{i+1} z_{i}\right)= \begin{cases}2 k+1+i, & \text { for } 1 \leq i \leq n-1 \\
2 k+1, & \text { for } i=n\end{cases} \\
w t\left(z_{i} z_{i+1}\right)= \begin{cases}3 k+1-i, & \text { for } 1 \leq i \leq n-3 \\
2 k+5+i, & \text { for } n-2 \leq i \leq n-1 \\
2 k+2+i, & \text { for } i=n, n \text { is odd } \\
2 k+1+i, & \text { for } i=n, n \text { is even }\end{cases} \\
\text { wt }\left(x_{i}\right)= \begin{cases}2+i, & \text { for } 1 \leq i \leq n-1 \\
2 n+2, & \text { for } i=n\end{cases} \\
\operatorname{wt}\left(z_{i}\right)= \begin{cases}3 k+2 n+2, & \text { for } i=1, n \text { is even } \\
3 k+2 n+3, & \text { for } i=1, n \text { is odd } \\
2 k+5 n+6-2 i, & \text { for } 2 \leq i \leq n-3 \\
2 k+4 n+7-i, & \text { for } n-2 \leq i \leq n\end{cases}
\end{gathered}
$$

Now, the weight of the edges and vertices of $T_{n}$ under the labeling $\phi$ are distinct. It is easy to check that there are no two edges of the same weight and there are no two vertices of the same weight. Thus, $\phi$ is a totally irregular total $k$-labeling. We conclude that $t s\left(T_{n}\right)=\left\lceil\frac{6 n+2}{3}\right\rceil$, which complete the proof.

\section{The Planar Graph $R_{n}$ (Pentagonal Circular Ladder)}

In [23], Bača defined the prism $D_{n}$ (Circular ladder) for $n \geq 3$. It is a cubic graph which can be defined as the cartesian product $P_{2} \times C_{n}$ on a path on two vertices with a cycle on $n$ vertices. Prism $D_{n}, n \geq 3$ is considered of $n$-cycle $y_{1}, y_{2}, y_{3}, \ldots, y_{n}$, an inner $n$-cycle $x_{1}, x_{2}, x_{3}, \ldots, x_{n}$, and a set of $n$ spokes $y_{i} z_{i}, i=1,2, \ldots, n, \mid V\left(D_{n}|=2 n| E,\left(D_{n} \mid=3 n\right.\right.$. The planar graph (pentagonal circular ladder) $R_{n}$ (see Figure 4 ) is obtained from the graph of prism $D_{n}$ by adding a new vertex $x_{i}$ between $y_{i}$ and $y_{i+1}$, for $i=1,2,3, \ldots, n$. The planar graph (pentagonal circular ladder) $R_{n}$ has

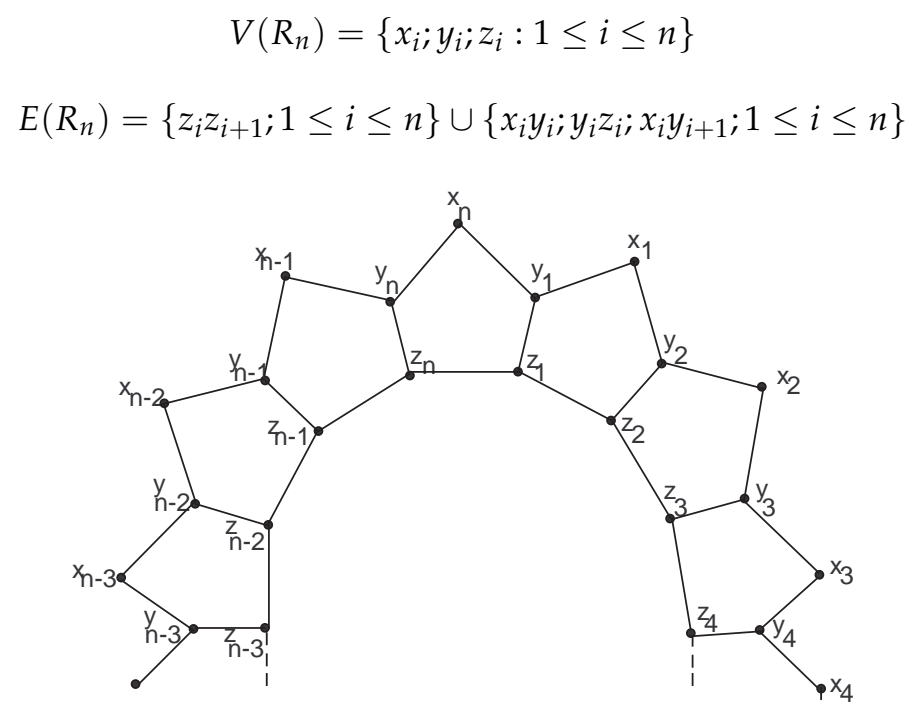

Figure 4. The planar graph $R_{n}$ (Pentagonal Circular Ladder). 
For our purpose, we call the cycle induced by $\left\{z_{i}: 1 \leq i \leq n\right\}$ the inner cycle, and the cycle induced by $\left\{y_{i}: 1 \leq i \leq n\right\} \cup\left\{x_{i}: 1 \leq i \leq n\right\}$ the outer cycle. All subscripts are taken under modulo $n$. In the next theorem, we determine the total irregularity strength of the planar graph $R_{n}$.

Theorem 4. Let $R_{n}, n \geq 4$ be a planar graph. Then, $t_{s}\left(R_{n}\right)=\left\lceil\frac{4 n+2}{3}\right\rceil$.

Proof. Since $\left|E\left(R_{n}\right)\right|=4 n$, from Theorem $1, \operatorname{tes}\left(R_{n}\right) \geq\left\lceil\frac{4 n+2}{3}\right\rceil$. In addition, $R_{n}$ has $n$ vertices of degree 2, $2 n$ vertices of degree 3; thus, from Theorem 2, we get $\operatorname{tvs}\left(T_{n}\right) \geq\left\lceil\frac{3 n+2}{4}\right\rceil$. From Equation (1), we get $t s\left(R_{n}\right) \geq\left\lceil\frac{4 n+2}{3}\right\rceil$. Now, we show that $t s\left(R_{n}\right) \leq\left\lceil\frac{4 n+2}{3}\right\rceil$. For this, we define a total labeling $\phi$ from $V\left(R_{n}\right) \cup E\left(R_{n}\right)$ into $\left\{1,2, \ldots,\left\lceil\frac{4 n+2}{3}\right\rceil\right\}$ and compute the vertex weight and edge weights in the following way.

Let $k=\left\lceil\frac{4 n+2}{3}\right\rceil$ and $1 \leq i \leq n$.

For $n=4$, we have,

$\phi\left(x_{i}\right)=i, \phi\left(y_{i}\right)=i, \phi\left(z_{i}\right)=k, \phi\left(x_{i} y_{i}\right)=1, \phi\left(x_{1} y_{2}\right)=1, \phi\left(x_{2} y_{3}\right)=1, \phi\left(x_{3} y_{4}\right)=1, \phi\left(x_{4} y_{1}\right)=6$, $\phi\left(y_{1} z_{1}\right)=5, \phi\left(y_{2} z_{2}\right)=2, \phi\left(y_{3} z_{3}\right)=4, \phi\left(y_{4} z_{4}\right)=4, \phi\left(z_{i} z_{i+1}\right)=2+i, w t\left(x_{i} y_{i}\right)=1+2 i, w t\left(x_{1} y_{2}\right)=4$, $w t\left(x_{2} y_{3}\right)=6, w t\left(x_{3} y_{4}\right)=8, w t\left(x_{4} y_{1}\right)=11, w t\left(x_{1}\right)=3, w t\left(x_{2}\right)=4, w t\left(x_{3}\right)=5, w t\left(x_{4}\right)=11$, $w t\left(y_{1}\right)=13, w t\left(y_{2}\right)=6, w t\left(y_{3}\right)=9, w t\left(y_{4}\right)=10, w t\left(z_{1}\right)=20, w t\left(z_{2}\right)=15, w t\left(z_{3}\right)=19$, $w t\left(z_{4}\right)=21$,

For $n=5$, we have, $\phi\left(x_{i}\right)=i, \phi\left(y_{i}\right)=i, \phi\left(z_{i}\right)=k-1, \phi\left(x_{i} y_{i}\right)=1, \phi\left(x_{1} y_{2}\right)=1, \phi\left(x_{2} y_{3}\right)=1, \phi\left(x_{3} y_{4}\right)=1, \phi\left(x_{4} y_{5}\right)=1$, $\phi\left(x_{5} y_{1}\right)=6, \phi\left(y_{1} z_{1}\right)=5, \phi\left(y_{2} z_{2}\right)=5, \phi\left(y_{3} z_{3}\right)=5, \phi\left(y_{4} z_{4}\right)=5, \phi\left(y_{5} z_{5}\right)=8, \phi\left(z_{1} z_{2}\right)=3$, $\phi\left(z_{2} z_{3}\right)=4, \phi\left(z_{3} z_{3}\right)=5, \phi\left(z_{4} z_{4}\right)=7, \phi\left(z_{5} z_{5}\right)=8, w t\left(x_{i} y_{i}\right)=1+2 i, w t\left(x_{i} y_{i+1}\right)=2+2 i, w t\left(x_{1}\right)=3$, $\omega t\left(x_{2}\right)=4, w t\left(x_{3}\right)=5, w t\left(x_{4}\right)=6, w t\left(x_{5} z\right)=12, w t\left(y_{1}\right)=13, w t\left(y_{2}\right)=9, w t\left(y_{3}\right)=10, w t\left(y_{4}\right)=11$, $w t\left(y_{5}\right)=15, w t\left(z_{1}\right)=23, w t\left(z_{2}\right)=19, w t\left(z_{3}\right)=21, w t\left(z_{4}\right)=24, w t\left(z_{5}\right)=30$,

For $n=7$, we have, $\phi\left(y_{i}\right)=i, \phi\left(z_{1}\right)=8, \phi\left(z_{2}\right)=9, \phi\left(z_{3}\right)=10, \phi\left(z_{4}\right)=11, \phi\left(z_{5}\right)=12, \phi\left(z_{6}\right)=13, \phi\left(z_{7}\right)=14$, $\phi\left(z_{8}\right)=14, \phi\left(z_{9}\right)=14, \phi\left(z_{10}\right)=14, \phi\left(z_{1} z_{2}\right)=7, \phi\left(z_{2} z_{3}\right)=7, \phi\left(z_{3} z_{4}\right)=7, \phi\left(z_{4} z_{5}\right)=7, \phi\left(z_{5} z_{6}\right)=7$, $\phi\left(z_{6} z_{7}\right)=12, \phi\left(z_{7} z_{8}\right)=13, \phi\left(z_{8} z_{9}\right)=14, \phi\left(z_{9} z_{10}\right)=12, \phi\left(z_{10} z_{1}\right)=12, w t\left(z_{1}\right)=41, w t\left(z_{2}\right)=37$, $w t\left(z_{3}\right)=38, w t\left(z_{4}\right)=39, w t\left(z_{5}\right)=40, w t\left(z_{6}\right)=46, w t\left(z_{7}\right)=53, w t\left(z_{8}\right)=55, w t\left(z_{9}\right)=54$, $w t\left(z_{10}\right)=52, \phi\left(y_{i} z_{i}\right)=k$,

$$
\begin{gathered}
\phi\left(x_{i}\right)= \begin{cases}i, & \text { for } 1 \leq i \leq n-1 \\
4, & \text { for } i=n\end{cases} \\
w t\left(x_{i}\right)= \begin{cases}2+i, & \text { for } 1 \leq i \leq n-1 \\
26, & \text { for } i=n\end{cases} \\
w t\left(y_{i}\right)= \begin{cases}30, & \text { for } i=1 \\
n+7+i, & \text { for } 2 \leq i \leq n-1 \\
31, & \text { for } i=n\end{cases} \\
\phi\left(x_{i} y_{i}\right)= \begin{cases}1, & \text { for } 1 \leq i \leq n-1 \\
k-n+2, & \text { for } i=n\end{cases} \\
\phi\left(x_{i} y_{i+1}\right)= \begin{cases}1, & \text { for } 1 \leq i \leq n-1 \\
k, & \text { for } i=n\end{cases}
\end{gathered}
$$




$$
\begin{gathered}
w t\left(x_{i} y_{i}\right)= \begin{cases}1+2 i, & \text { for } 1 \leq i \leq n-1 \\
22, & \text { for } i=n\end{cases} \\
w t\left(x_{i} y_{i+1}\right)= \begin{cases}2+2 i, & \text { for } 1 \leq i \leq n-1 \\
21, & \text { for } i=n\end{cases} \\
w t\left(y_{i} z_{i}\right)= \begin{cases}2 i+2 k-\frac{2(n-1)}{3}-1, & \text { for } 1 \leq i \leq \frac{k}{2} \\
2 k+1+i, & \text { for } \frac{k+2}{2} \leq i \leq n\end{cases} \\
w t\left(z_{i} z_{i+1}\right)= \begin{cases}2 n+2+2 i, & \text { for } 1 \leq i \leq 5 \\
4 n-1, & \text { for } i=6 \\
k+2 n+i, & \text { for } i=7,8 \\
4 n, & \text { for } i=9 \\
3 n+4, & \text { for } i=10\end{cases}
\end{gathered}
$$

For $n=10$, we have,

$\phi\left(y_{i}\right)=i, \phi\left(z_{1}\right)=6, \phi\left(z_{2}\right)=7, \phi\left(z_{3}\right)=8, \phi\left(z_{4}\right)=9, \phi\left(z_{5}\right)=10, \phi\left(z_{6}\right)=10, \phi\left(z_{7}\right)=10, \phi\left(z_{1} z_{2}\right)=5$,
$\phi\left(z_{2} z_{3}\right)=5, \phi\left(z_{3} z_{4}\right)=5, \phi\left(z_{4} z_{5}\right)=10, \phi\left(z_{5} z_{6}\right)=10, \phi\left(z_{6} z_{7}\right)=8, \phi\left(z_{7} z_{1}\right)=8, w t\left(x_{1}\right)=3$,
$\operatorname{wt}\left(x_{2}\right)=4, w t\left(x_{3}\right)=5, w t\left(x_{4}\right)=6, w t\left(x_{5} z\right)=7, w t\left(x_{6} z\right)=8, w t\left(x_{7}\right)=19, w t\left(y_{1}\right)=22, w t\left(y_{2}\right)=14$,
$\operatorname{wt}\left(y_{3}\right)=15, w t\left(y_{4}\right)=16, w t\left(y_{5}\right)=17, w t\left(y_{6} z\right)=18, w t\left(x_{7}\right)=23, w t\left(z_{1}\right)=29, w t\left(z_{2}\right)=27$,
$w t\left(z_{3}\right)=28, w t\left(z_{4}\right)=34, w t\left(z_{5}\right)=40, w t(z)=38, w t\left(z_{7}\right)=36, \phi\left(y_{i} z_{i}\right)=k$,

$$
\begin{gathered}
\phi\left(x_{i}\right)= \begin{cases}i, & \text { for } 1 \leq i \leq n-1 \\
6, & \text { for } i=n\end{cases} \\
\phi\left(x_{i} y_{i}\right)= \begin{cases}1, & \text { for } 1 \leq i \leq n-1 \\
k-n+2, & \text { for } i=n\end{cases} \\
\phi\left(x_{i} y_{i+1}\right)= \begin{cases}1, & \text { for } 1 \leq i \leq n-1 \\
k, & \text { for } i=n\end{cases} \\
w w t\left(x_{i} y_{i}\right)= \begin{cases}1+2 i, & \text { for } 1 \leq i \leq n-1 \\
16, & \text { for } i=n\end{cases} \\
w t\left(x_{i} y_{i+1}\right)= \begin{cases}2+2 i, & \text { for } 1 \leq i \leq n-1 \\
15, & \text { for } i=n\end{cases} \\
w t\left(z_{i} z_{i+1}\right)= \begin{cases}2 i+2 k-\frac{2 n}{3}-1, & \text { for } 1 \leq i \leq \frac{k+2}{2} \\
2 k+i, & \text { for } \frac{k+4}{2} \leq i \leq n \\
k+2 n+4, & \text { for } 1 \leq i \leq \frac{k}{2}-2 \\
k+2 n, & \text { for } i=n-1 \leq n-2\end{cases}
\end{gathered}
$$

For $n \geq 6$ and $n \neq 7,10$, we have $\phi\left(y_{i}\right)=i, \phi\left(y_{i} z_{i}\right)=k$,

$$
\begin{gathered}
\phi\left(x_{i} y_{i}\right)= \begin{cases}1, & \text { for } 1 \leq i \leq n-1 \\
k-n+2, & \text { for } i=n\end{cases} \\
\phi\left(x_{i} y_{i+1}\right)= \begin{cases}1, & \text { for } 1 \leq i \leq n-1 \\
k, & \text { for } i=n\end{cases}
\end{gathered}
$$


Case 1. when $n \equiv 0(\bmod 3)$

$$
\begin{aligned}
& \phi\left(x_{i}\right)= \begin{cases}i, & \text { for } 1 \leq i \leq n-1 \\
\frac{k-3}{2}, & \text { for } i=n\end{cases} \\
& \phi\left(z_{i}\right)= \begin{cases}k-\frac{2 n}{3}-1+i, & \text { for } 1 \leq i \leq \frac{k+1}{2} \\
k, & \text { for } \frac{k+3}{2} \leq i \leq n\end{cases} \\
& \phi\left(z_{i} z_{i+1}\right)= \begin{cases}\frac{k+1}{2} & \text { for } 1 \leq i \leq \frac{k+1}{2}-2 \\
n+2, & \text { for } i=\frac{k+1}{2}-1 \\
k-n+i, & \text { for } \frac{k+1}{2} \leq i \leq n-1 \\
k-1, & \text { for } i=n\end{cases} \\
& w t\left(x_{i}\right)= \begin{cases}i+2, & \text { for } 1 \leq i \leq n-1 \\
3 k-\frac{5 n}{3}, & \text { for } i=n\end{cases} \\
& w t\left(y_{i}\right)= \begin{cases}2 k+2, & \text { for } i=1 \\
4(k-n)-1+i, & \text { for } 2 \leq i \leq n-1 \\
2 k+3, & \text { for } i=n\end{cases} \\
& w t\left(z_{i}\right)= \begin{cases}\frac{7(k+1)}{2}-\frac{2 n}{3}-4, & \text { for } i=1 \\
3 k-\frac{2 n}{3}+i, & \text { for } 2 \leq i \leq \frac{k-3}{2} \\
\frac{5(k+1)}{2}+n-1, & \text { for } i=\frac{k-1}{2} \\
3 k+2+i, & \text { for } \frac{k+1}{2} \leq i \leq \frac{k+3}{2} \\
4(k-1)-2 n+3+2 i, & \frac{k+5}{2} \leq i \leq n-1 \\
4 k-2, & \text { for } i=n\end{cases} \\
& w t\left(x_{i} y_{i}\right)= \begin{cases}2 i+1, & \text { for } 1 \leq i \leq n-1 \\
2 k-\frac{2 n}{3}, & \text { for } i=n\end{cases} \\
& w t\left(x_{i} y_{i+1}\right)= \begin{cases}2 i+2, & \text { for } 1 \leq i \leq n-1 \\
2 k-\frac{2 n}{3}-1, & \text { for } i=n\end{cases} \\
& w t\left(y_{i} z_{i}\right)= \begin{cases}2 i+2 k-\frac{2 n}{3}-1, & \text { for } 1 \leq i \leq \frac{k+1}{2}-1 \\
2 k+i, & \text { for } \frac{k+1}{2} \leq i \leq n\end{cases} \\
& w t\left(z_{i} z_{i+1}\right)= \begin{cases}2 n+2+2 i, & \text { for } 1 \leq i \leq \frac{k+1}{2}-2 \\
3 n+3+i, & \text { for } \frac{k+1}{2}-1 \leq i \leq n-1 \\
k+2 n+1, & \text { for } i=n\end{cases}
\end{aligned}
$$

Case 2. when $n \equiv 1(\bmod 3)$

$$
\begin{gathered}
\phi\left(x_{i}\right)= \begin{cases}i, & \text { for } 1 \leq i \leq n-1 \\
k-\frac{(2 n+1)}{3}-1, & \text { for } i=n\end{cases} \\
\phi\left(z_{i}\right)= \begin{cases}k-\frac{2 n+1}{3}+i, & \text { for } 1 \leq i \leq \frac{k}{2} \\
k, & \text { for } \frac{k}{2}+1 \leq i \leq n\end{cases} \\
\phi\left(z_{i} z_{i+1}\right)= \begin{cases}\frac{k}{2}, & \text { for } 1 \leq i \leq \frac{k}{2}-2 \\
n+2, & \text { for } i=\frac{k}{2}-1 \\
k-n+1+i, & \text { for } \frac{k}{2} \leq i \leq n-1 \\
k-2, & \text { for } i=n\end{cases}
\end{gathered}
$$




$$
\begin{aligned}
& w t\left(x_{i}\right)= \begin{cases}i+2, & \text { for } 1 \leq i \leq n-1 \\
3 k-\frac{5 n+1}{3}+1, & \text { for } i=n\end{cases} \\
& w t\left(y_{i}\right)= \begin{cases}2 k+2, & \text { for } i=1 \\
4(k-n)+i, & \text { for } 2 \leq i \leq n-1 \\
2 k+3, & \text { for } i=n\end{cases} \\
& w t\left(z_{i}\right)= \begin{cases}\frac{7 k}{2}-\frac{2 n+1}{3}-1, & \text { for } i=1 \\
3 k-\frac{2 n+1}{3}+i, & \text { for } 2 \leq i \leq \frac{k}{2}-2 \\
\frac{5 k}{2}+n+1, & \text { for } i=\frac{k}{2}-1 \\
3 k+3+i, & \text { for } \frac{k}{2} \leq i \leq \frac{k}{2}+1 \\
4 k-2 n+1+2 i, & \text { for } \frac{k}{2}+2 \leq i \leq n-1 \\
4 k-2, & \text { for } i=n\end{cases} \\
& w t\left(x_{i} y_{i}\right)= \begin{cases}2 i+1, & \text { for } 1 \leq i \leq n-1 \\
2 k-\frac{2 n+1}{3}+1, & i=n .\end{cases} \\
& w t\left(x_{i} y_{i+1}\right)= \begin{cases}2 i+2, & 1 \leq i \leq n-1 \\
2 k-\frac{2 n+1}{3}, & \text { for } i=n\end{cases} \\
& w t\left(y_{i} z_{i}\right)= \begin{cases}2 i+2 k-\frac{2 n+1}{3}, & \text { for } 1 \leq i \leq \frac{k}{2} \\
2 k+i, & \text { for } \frac{k}{2}+1 \leq i \leq n\end{cases} \\
& w t\left(z_{i} z_{i+1}\right)= \begin{cases}2 n+2+2 i, & \text { for } 1 \leq i \leq \frac{k}{2}-2 \\
3 n+3+i, & \text { for } \frac{k}{2}-1 \leq i \leq n-1 \\
k+2 n, & \text { for } i=n\end{cases}
\end{aligned}
$$

Case 3. when $n \equiv 2(\bmod 3)$

$$
\begin{gathered}
\phi\left(x_{i}\right)= \begin{cases}i, & \text { for } 1 \leq i \leq n-1 \\
\frac{k-4}{2}, & \text { for } i=n\end{cases} \\
\phi\left(z_{i}\right)= \begin{cases}k-\frac{2 n+2}{3}-1+i, & \text { for } 1 \leq i \leq \frac{k+2}{2} \\
k, & \text { for } \frac{k+4}{2} \leq i \leq n\end{cases} \\
\phi\left(z_{i} z_{i+1}\right)= \begin{cases}\frac{k}{2}+1, & \text { for } 1 \leq i \leq \frac{k}{2}-1 \\
n+2, & \text { for } i=\frac{k}{2} \\
k-n-1+i, & \text { for } \frac{k}{2}+1 \leq i \leq n-1 \\
k, & \text { for } i=n\end{cases} \\
w t\left(x_{i}\right)= \begin{cases}i+2, & \text { for } 1 \leq i \leq n-1 \\
3 k-\frac{5 n+5}{3}+1, & \text { for } i=n\end{cases} \\
w t\left(y_{i}\right)= \begin{cases}2 k+2, & \text { for } i=1 \\
4(k-n)-2+i, & \text { for } 2 \leq i \leq n-1 \\
2 k+3, & \text { for } i=n\end{cases} \\
\text { wt }\left(z_{i}\right)= \begin{cases}\frac{7 k}{2}-\frac{2(n+1)}{3}+1, & \text { for } i=1 \\
3 k-\frac{2(n+1)}{3}+1+i, & \text { for } 2 \leq i \leq \frac{k}{2}-1 \\
\frac{5 k}{2}+n+2, & \text { for } i=\frac{k}{2} \\
3 k+2+i, & \text { for } \frac{k}{2}+1 \leq i \leq \frac{k}{2}+2 \\
4 k-2 n-1+2 i, & \text { for } \frac{k}{2}+3 \leq i \leq n-1 \\
4 k-2, & \text { for } i=n\end{cases}
\end{gathered}
$$




$$
\begin{gathered}
w t\left(x_{i} y_{i}\right)= \begin{cases}2 i+1, & \text { for } 1 \leq i \leq n-1 \\
2 k+\frac{2 n+2}{3}, & \text { for } i=n\end{cases} \\
w t\left(x_{i} y_{i+1}\right)= \begin{cases}2 i+2, & \text { for } 1 \leq i \leq n-1 \\
2 k-\frac{2 n+2}{3}-1, & \text { for } i=n\end{cases} \\
w t\left(y_{i} z_{i}\right)= \begin{cases}2 i+2 k-\frac{2 n+2}{3}-1, & \text { for } 1 \leq i \leq \frac{k}{2} \\
2 k+i, & \text { for } \frac{k}{2}+1 \leq i \leq n\end{cases} \\
w t\left(z_{i} z_{i+1}\right)= \begin{cases}2 n+2+2 i, & \text { for } 1 \leq i \leq \frac{k}{2}-1 \\
3 n+3+i, & \text { for } \frac{k}{2} \leq i \leq n-1 \\
k+2 n+2, & \text { for } i=n\end{cases}
\end{gathered}
$$

The weight of the edges and vertices under the labeling $\phi$ are distinct. It is easy to check that there are no two edges of the same weight and there are no two vertices of the same weight. Thus, $\phi$ is a totally irregular total $k$-labeling. We conclude that $t s\left(R_{n}\right)=\left\lceil\frac{4 n+2}{3}\right\rceil$, which complete the proof.

\section{The Planar Graph $Q_{n}$}

In [23], Bača defined the planar graph (pentagonal circular ladder) $R_{n}$. The planar graph $Q_{n}$ (see Figure 5) is obtained from the planar graph (pentagonal circular ladder) $R_{n}$ by adding new edges $y_{i} y_{i+1}, z_{i} w_{i}, w_{i} w_{i+1}$. The planar graph $Q_{n}$ has

$$
\begin{gathered}
V\left(Q_{n}\right)=\left\{x_{i} ; y_{i} ; z_{i} ; w_{i}: 1 \leq i \leq n\right\} \\
E\left(Q_{n}\right)=\left\{y_{i} y_{i+1} ; w_{i} w_{i+1} ; z_{i} z_{i+1} ; x_{i} y_{i} ; x_{i} y_{i+1} ; y_{i} z_{i} ; z_{i} w_{i}: 1 \leq i \leq n\right\}
\end{gathered}
$$

The planar graph $Q_{n}$ has $4 n$ vertices and $7 n$ edges. For our purpose, we call the cycle induced by $\left\{w_{i}: 1 \leq i \leq n\right\}$ the inner cycle, the cycle induced by $\left\{z_{i}: 1 \leq i \leq n\right\}$ the middle cycle, the cycle induced by $\left\{y_{i}: 1 \leq i \leq n\right\}$ the outer cycle, and the set of vertices $\left\{x_{i}: 1 \leq i \leq n\right\}$ the set of outer vertices. The subscript $n+1$ must be replaced by 1 .

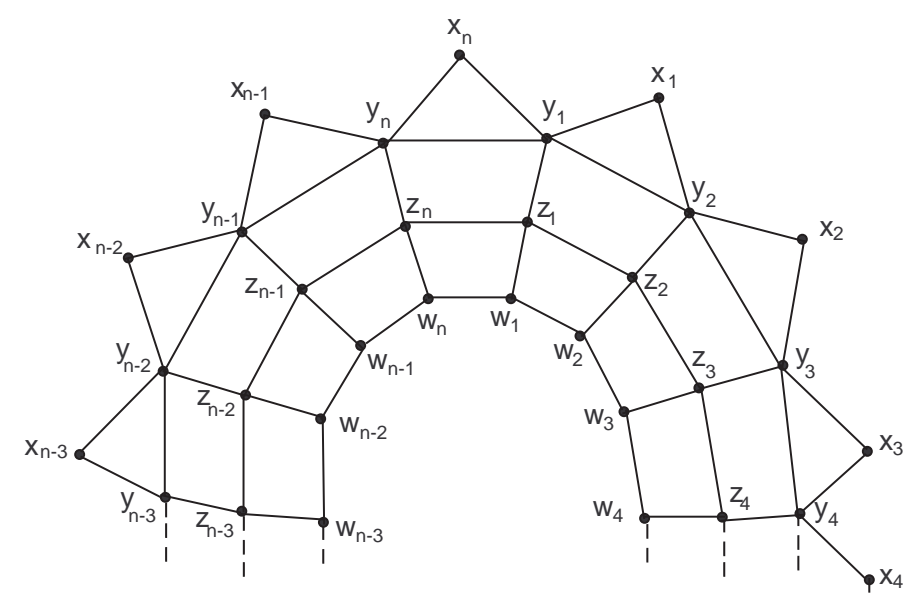

Figure 5. The planar graph $Q_{n}$.

Theorem 5. Let $Q_{n}, n \geq 4$ be a planar graph. Then, $t s\left(Q_{n}\right)=\left\lceil\frac{7 n+2}{3}\right\rceil$.

Proof. Since $\left|E\left(Q_{n}\right)\right|=7 n$, from Theorem 1 tes $\left(Q_{n}\right) \geq\left\lceil\frac{7 n+2}{3}\right\rceil$. In addition, $Q_{n}$ has $n$ vertices of degree 2, $n$ vertices of degree 3, $n$ vertices of degree 4 and $n$ vertices of degree 5 ; thus, from Theorem 2 , we get $\operatorname{tvs}\left(Q_{n}\right) \geq\left\lceil\frac{4 n+2}{6}\right\rceil$. From Equation (1), we get $t s\left(Q_{n}\right) \geq\left\lceil\frac{7 n+2}{3}\right\rceil$. Now, we show that $t s\left(Q_{n}\right) \leq\left\lceil\frac{7 n+2}{3}\right\rceil$. 
For this, we define a total labeling $\phi$ from $V\left(Q_{n}\right) \cup E\left(Q_{n}\right)$ into $\left\{1,2, \ldots,\left\lceil\frac{7 n+2}{3}\right\rceil\right\}$ and compute the vertex weight and edge weights in the following way.

Let $k=\left\lceil\frac{7 n+2}{3}\right\rceil$ and $1 \leq i \leq n$,

$\phi\left(x_{i}\right)=i, \phi\left(y_{i}\right)=i, \phi\left(z_{i}\right)=k, \phi\left(x_{i} y_{i}\right)=1, w t\left(x_{i} y_{i}\right)=1+2 i, w t\left(x_{i} y_{i+1}\right)=2+2 i$, $w t\left(y_{i} z_{i}\right)=4 n+2+i, w t\left(y_{i} y_{i+1}\right)=2 n+2+i, w t\left(w_{i} w_{i+1}\right)=3 n+2+i$,

$$
\begin{gathered}
\phi\left(x_{i} y_{i+1}\right)= \begin{cases}1, & \text { for } 1 \leq i \leq n-1 \\
n+1, & \text { for } i=n\end{cases} \\
\phi\left(y_{i} y_{i+1}\right)= \begin{cases}2 n+1-i, & \text { for } 1 \leq i \leq n-1 \\
2 n+1, & \text { for } i=n\end{cases} \\
\phi\left(w_{i} w_{i+1}\right)= \begin{cases}i, & \text { for } 1 \leq i \leq n, n \text { is even } \\
i+1, & \text { for } 1 \leq i \leq n, n \text { is odd }\end{cases} \\
w t\left(x_{i}\right)= \begin{cases}2+i, & \text { for } 1 \leq i \leq n-1 \\
2 n+2, & \text { for } i=n\end{cases}
\end{gathered}
$$

Case 1. when $n \equiv 0(\bmod 6)$ and $1 \leq i \leq n$

$\phi\left(w_{i}\right)=\frac{3 n}{2}+1, \phi\left(y_{i} z_{i}\right)=\frac{5 n}{3}+1, \phi\left(z_{i} z_{i+1}\right)=\frac{4 n}{3}+i, w t\left(z_{i} z_{i+1}\right)=2 k+\frac{4 n}{3}+i, w t\left(z_{i}\right)=\frac{53 n}{6}+3+i$, $w t\left(w_{i}\right)=\frac{11 n}{3}+2+i$

$$
\begin{gathered}
\phi\left(z_{i} w_{i}\right)= \begin{cases}\frac{7 n}{6}+1, & \text { for } i=1 \\
\frac{13 n}{6}+2-i, & \text { for } 2 \leq i \leq n\end{cases} \\
w t\left(z_{i} w_{i}\right)= \begin{cases}k+\frac{8 n}{3}+2, & \text { for } i=1 \\
k+\frac{11 n}{3}+3-i, & \text { for } 2 \leq i \leq n\end{cases} \\
w t\left(y_{i}\right)= \begin{cases}\frac{20 n}{3}+5, & \text { for } i=1 \\
\frac{17 n}{3}+6-i, & \text { for } 2 \leq i \leq n-1 \\
\frac{17 n}{3}+6, & \text { for } i=n\end{cases}
\end{gathered}
$$

Case 2. when $n \equiv 1(\bmod 6)$ and $1 \leq i \leq n$

$$
\begin{gathered}
\phi\left(w_{i}\right)=\frac{3(n-1)}{2}+2, \phi\left(y_{i} z_{i}\right)=\frac{5(n-1)}{3}+3, \phi\left(z_{i} z_{i+1}\right)=\frac{4(n-1)}{3}+2+i, w t\left(z_{i} z_{i+1}\right)=2 k+\frac{4(n-1)}{3}+2+i, \\
w t\left(z_{i}\right)=\frac{53(n-1)}{6}+14+i, w t\left(w_{i}\right)=\frac{11(n-1)}{3}+8+i, \\
\phi\left(z_{i} w_{i}\right)= \begin{cases}\frac{7(n-1)}{6}+3, & \text { for } i=1 \\
\frac{13(n-1)}{6}+5-i, & \text { for } 2 \leq i \leq n\end{cases} \\
w t\left(z_{i} w_{i}\right)= \begin{cases}k+\frac{8(n-1)}{3}+5, & \text { for } i=1 \\
k+\frac{11(n-1)}{3}+7-i, & \text { for } 2 \leq i \leq n\end{cases} \\
w t\left(y_{i}\right)= \begin{cases}\frac{20(n-1)}{3}+12, & \text { for } i=1 \\
\frac{17(n-1)}{3}+12-i, & \text { for } 2 \leq i \leq n-1 \\
\frac{17(n-1)}{3}+12, & \text { for } i=n\end{cases}
\end{gathered}
$$

Case 3. when $n \equiv 2(\bmod 6)$ and $1 \leq i \leq n$ 

$\phi\left(w_{i}\right)=\frac{3(n-2)}{2}+4, \phi\left(y_{i} z_{i}\right)=\frac{5(n-2)}{3}+4, \phi\left(z_{i} z_{i+1}\right)=\frac{4(n-2)}{3}+2+i, w t\left(z_{i} z_{i+1}\right)=2 k+\frac{4(n-2)}{3}+2+i$,
$w t\left(z_{i}\right)=\frac{53(n-2)}{6}+19+i, w t\left(w_{i}\right)=\frac{11(n-2)}{3}+9+i$,

$$
\begin{gathered}
\phi\left(z_{i} w_{i}\right)= \begin{cases}\frac{7(n-2)}{6}+3, & \text { for } i=1 \\
\frac{13(n-2)}{6}+6-i, & \text { for } 2 \leq i \leq n\end{cases} \\
w t\left(z_{i} w_{i}\right)= \begin{cases}k+\frac{8(n-2)}{3}+7, & \text { for } i=1 \\
k+\frac{11(n-2)}{3}+10-i, & \text { for } 2 \leq i \leq n\end{cases} \\
w t\left(y_{i}\right)= \begin{cases}\frac{20(n-2)}{3}+18, & \text { for } i=1 \\
\frac{17(n-2)}{3}+17-i, & \text { for } 2 \leq i \leq n-1 \\
\frac{17(n-2)}{3}+17, & \text { for } i=n\end{cases}
\end{gathered}
$$

Case 4. when $n \equiv 3(\bmod 6)$ and $1 \leq i \leq n$

$\phi\left(w_{i}\right)=\frac{3(n-3)}{2}+5, \phi\left(y_{i} z_{i}\right)=\frac{5(n-3)}{3}+6, \phi\left(z_{i} z_{i+1}\right)=\frac{4(n-2)}{3}+4+i, w t\left(z_{i} z_{i+1}\right)=2 k+\frac{4(n-3)}{3}+4+i$, $w t\left(z_{i}\right)=\frac{53(n-3)}{6}+30+i, w t\left(w_{i}\right)=\frac{11(n-3)}{3}+15+i$,

$$
\begin{gathered}
\phi\left(z_{i} w_{i}\right)= \begin{cases}\frac{7(n-3)}{6}+5, & \text { for } i=1 \\
\frac{13(n-3)}{6}+9-i, & \text { for } 2 \leq i \leq n\end{cases} \\
w t\left(z_{i} w_{i}\right)= \begin{cases}k+\frac{8(n-3)}{3}+10, & \text { for } i=1 \\
k+\frac{11(n-3)}{3}+14-i, & \text { for } 2 \leq i \leq n\end{cases} \\
w t\left(y_{i}\right)= \begin{cases}\frac{20(n-3)}{3}+25, & \text { for } i=1 \\
\frac{17(n-3)}{3}+23-i, & \text { for } 2 \leq i \leq n-1 \\
\frac{17(n-3)}{3}+23, & \text { for } i=n\end{cases}
\end{gathered}
$$

Case 5. when $n \equiv 4(\bmod 6)$ and $1 \leq i \leq n$

$\phi\left(w_{i}\right)=\frac{3(n-4)}{2}+7, \phi\left(y_{i} z_{i}\right)=\frac{5(n-4)}{3}+8, \phi\left(z_{i} z_{i+1}\right)=\frac{4(n-4)}{3}+6+i, w t\left(z_{i} z_{i+1}\right)=2 k+\frac{4(n-4)}{3}+6+i$, $w t\left(z_{i}\right)=\frac{53(n-4)}{6}+40+i, w t\left(w_{i}\right)=\frac{11(n-4)}{3}+17+i$,

$$
\begin{gathered}
\phi\left(z_{i} w_{i}\right)= \begin{cases}\frac{7(n-4)}{6}+6, & \text { for } i=1 \\
\frac{13(n-4)}{6}+11-i, & \text { for } 2 \leq i \leq n\end{cases} \\
w t\left(z_{i} w_{i}\right)= \begin{cases}k+\frac{8(n-4)}{3}+13, & \text { for } i=1 \\
k+\frac{11(n-4)}{3}+18-i, & \text { for } 2 \leq i \leq n\end{cases} \\
w t\left(y_{i}\right)= \begin{cases}\frac{20(n-4)}{3}+32, & \text { for } i=1 \\
\frac{17(n-4)}{3}+29-i, & \text { for } 2 \leq i \leq n-1 \\
\frac{17(n-4)}{3}+29, & \text { for } i=n\end{cases}
\end{gathered}
$$

Case 6. when $n \equiv 5(\bmod 6)$ and $1 \leq i \leq n$

$\phi\left(w_{i}\right)=\frac{3(n-5)}{2}+8, \phi\left(y_{i} z_{i}\right)=\frac{5(n-5)}{3}+9, \phi\left(z_{i} z_{i+1}\right)=\frac{4(n-5)}{3}+6+i, w t\left(z_{i} z_{i+1}\right)=2 k+\frac{4(n-5)}{3}+6+i$, $w t\left(z_{i}\right)=\frac{53(n-5)}{6}+46+i, w t\left(w_{i}\right)=\frac{11(n-5)}{3}+22+i$,

$$
\phi\left(z_{i} w_{i}\right)= \begin{cases}\frac{7(n-5)}{6}+7, & \text { for } i=1 \\ \frac{13(n-5)}{6}+13-i, & \text { for } 2 \leq i \leq n\end{cases}
$$




$$
\begin{gathered}
w t\left(z_{i} w_{i}\right)= \begin{cases}k+\frac{8(n-5)}{3}+15, & \text { for } i=1 \\
k+\frac{11(n-5)}{3}+21-i, & \text { for } 2 \leq i \leq n\end{cases} \\
w t\left(y_{i}\right)= \begin{cases}\frac{20(n-5)}{3}+38, & \text { for } i=1 \\
\frac{17(n-5)}{3}+34-i, & \text { for } 2 \leq i \leq n-1 \\
\frac{17(n-5)}{3}+34, & \text { for } i=n\end{cases}
\end{gathered}
$$

The weight of the edges and vertices of $S_{n}$ under the labeling $\phi$ are distinct. It is easy to check that there are no two edges of the same weight and there are no two vertices of the same weight. Thus, $\phi$ is a totally irregular total $k$-labeling. We conclude that $t s\left(Q_{n}\right)=\left\lceil\frac{7 n+2}{3}\right\rceil$, which completes the proof.

\section{Conclusions}

In this paper, we discus the total edge irregular $k$ labeling, total vertex irregular $k$ labeling and totally irregular total $k$ labeling of planar graphs. We provide exact result of total irregularity strength ts for the planar graph $T_{n}$, the planar graph $R_{n}$ (Pentagonal Circular Ladder) and the planar graph $Q_{n}$. In the future, we are interested in computing the total irregularity strength $t s$ for the other planar graphs.

Author Contributions: H.Y. contribute for supervision, project administration, funding and analyzed the data curation. M.K.S. contribute for designing the experiments, validation, conceptualization. M.I. contribute for formal analysing experiments, resources, software and some computations. S.A and A.A. contribute for Investigation, Methodology and wrote the initial draft of the paper. M.K.S. investigated the initial draft and wrote the final draft. All authors read and approved the final version of the paper.

Funding: This work was supported by the Soft Scientific Research Foundation of Sichuan Province under grant 2018ZR0265, Project of Sichuan Military and Civilian Integration Strategy Research Center under grant JMRH-1818, and Key Project of Sichuan Provincial Department of Education under grant 18ZA0118.

Acknowledgments: The authors are grateful to the anonymous referees for their valuable comments and suggestions that improved this paper.

Conflicts of Interest: The authors declare no conflict of interest.

\section{References}

1. Gallian, J. A dynamic survey of graph labeling. Electron. J. Comb. 2009, 16, 1-442.

2. Chartrand, G.; Jacobson, M.S.; Lehel, J.; Oellermann, O.R.; Ruiz, S.; Saba, F. Irregular networks. Congr. Numer. 1988, 64, 187-192.

3. Bača, M.; Jendrol, S.; Miller, M.; Ryan, J. On irregular total labellings. Discret. Math. 2007, 307, $1378-1388$. [CrossRef]

4. Ivančo, J.; Jendrol, S. Total edge irregularity strength of trees. Discuss. Math. Graph Theory 2006, 26, 449-456.

5. Nurdin; Baskoro, E.T.; Salman, A.N.M.; Gaos, N.N. On total vertex irregularity strength of trees. Discret. Math. 2010, 310, 3043-3048. [CrossRef]

6. Jendrol', S.; Miškuf, J.; Soták, R. Total edge irregularity strength of complete graphs and complete bipartite graphs. Electron. Notes Discret. Math. 2007, 28, 281-285. [CrossRef]

7. Jendrol', S.; Miškuf, J.; Soták, R. Total edge irregularity strength of complete graphs and complete bipartite graphs. Discret. Math. 2010, 310, 400-407. [CrossRef]

8. Al-Mushayt, O.; Ahmad, A.; Siddiqui, M.K. On the total edge irregularity strength of hexagonal grid graphs. Australas. J. Comb. 2012, 53, 263-271.

9. Chunling, T.; Xiaohui, L.; Yuansheng, Y.; Liping, W. Irregular total labellings of grids. Utilitas Math. 2010, 81, 3-13.

10. Bača, M.; Siddiqui, M.K. Total edge irregularity strength of generalized prism. Appl. Math. Comput. 2014, 235, 168-173. [CrossRef]

11. Ahmad, A.; Al Mushayt, O.; Siddiqui, M.K. Total edge irregularity strength of strong product of cycles and paths. UPB Sci. Bull. Ser. A 2014, 76, 147-156.

12. Ahmad, A.; Bača, M.; Siddiqui, M.K. On edge irregular total labeling of categorical product of two cycles. Theory Comp. Syst. 2014, 54,1-12. [CrossRef] 
13. Ahmad, A.; Siddiqui, M.K.; Afzal, D. On the total edge irregularity strength of zigzag graphs. Australas. J. Comb. 2012, 54, 141-149.

14. Ahmad, A.; Bača, M.; Bashir, Y.; Siddiqui, M.K. Total edge irregularity strength of strong product of two paths. Ars Comb. 2012, 106, 449-459.

15. Anholcer, M.; Palmer, C. Irregular labelings of circulant graphs. Discret. Math. 2012, 312, 3461-3466. [CrossRef]

16. Marzuki, C.C.; Salman, A.N.M.; Miller, M. On the total irregularity strength on cycles and paths. Far East J. Math. Sci. 2013, 82, 1-21.

17. Ramdani, R.; Salman, A.N.M. On the total irregularity strength of some cartesian product graphs. AKCE Int. J. Graphs Comb. 2013, 10, 199-209.

18. Bača, M.; Siddiqui, M.K. On total edge irregularity strength of strong product of two cycles. Utilitas Math. 2017, 104, 255-275.

19. Siddiqui, M.K.; Afzal, D.; Faisal, M.R. Total edge irregularity strength of accordion graphs. J. Comb. Optim. 2017, 34, 534-544. [CrossRef]

20. Siddiqui, M.K.; Miller, M.; Ryan, J. Total edge irregularity strength of octagonal grid graph. Utilitas Math. 2017, 103, 277-287.

21. Ahmad, A.; Siddiqui, M.K.; Ibrahim, M.; Asif, M. On the total irregularity strength of Generalized Petersen Graph. Math. Rep. 2016, 68, 139-147.

22. Siddiqui, M.K. On irregularity strength of convex polytope graphs with certain pendent edges added. Ars Comb. 2016, 129, 199-210.

23. Bača, M. Labeling of two classes of convex polytopes. Utilitas Math. 1998, 34, 24-31.

(C) 2018 by the authors. Licensee MDPI, Basel, Switzerland. This article is an open access article distributed under the terms and conditions of the Creative Commons Attribution (CC BY) license (http:// creativecommons.org/licenses/by/4.0/). 